Research Article

\title{
Single-Nucleotide Polymorphisms in XPO5 are Associated with Noise-Induced Hearing Loss in a Chinese Population
}

\author{
Ning Wang, ${ }^{1,2}$ Boshen Wang $\mathbb{D}^{1,},{ }^{1,2}$ Jiadi Guo, ${ }^{2,3}$ Suhao Zhang $\left(\mathbb{D},{ }^{4}\right.$ Lei Han, ${ }^{2}$ Juan Zhang $\mathbb{D}^{1}{ }^{1}$ \\ and Baoli Zhu $\mathbb{i D}^{1,2,3}$ \\ ${ }^{1}$ Key Laboratory of Environmental Medicine Engineering of Ministry of Education, School of Public Health, Southeast University, \\ Nanjing 210009, Jiangsu, China \\ ${ }^{2}$ Jiangsu ProvincialCenter for Disease Prevention and Control, Nanjing 210009, Jiangsu, China \\ ${ }^{3}$ Center for Global Health, School of Public Health, Nanjing Medical University, Nanjing 210000, Jiangsu, China \\ ${ }^{4}$ School of Public Health, NantongUniversity, Nantong 226000, Jiangsu, China \\ Correspondence should be addressed to Baoli Zhu; zhbl5888@sina.com
}

Received 21 May 2019; Revised 20 December 2019; Accepted 30 December 2019; Published 17 February 2020

Academic Editor: Tzi Bun Ng

Copyright (c) 2020 Ning Wang et al. This is an open access article distributed under the Creative Commons Attribution License, which permits unrestricted use, distribution, and reproduction in any medium, provided the original work is properly cited.

\begin{abstract}
Objectives. The purpose of this study was to investigate the correlation between single-nucleotide polymorphism (SNP) in $3^{\prime} \mathrm{UTR}$ of XPO5 gene and the occurrence of noise-induced hearing loss (NIHL), and to further explore the regulatory mechanism of miRNAs in NIHL on XPO5 gene. Methods. We conducted a case-control study involving 1040 cases and 1060 controls. The effects of SNPs on XPO5 expression were studied by genotyping, real-time polymerase chain reaction (qPCR), cell transfection, and the dual-luciferase reporter assay. Results. We genotyped four SNPs (rs2257082, rs11077, rs7755135, and rs1106841) in the XPO5 gene. The rs2257082 AG/GG carriers have special connection to an increased risk of noise-induced hearing loss compared to the AA carriers. The rs11077TG/GG carriers had a significantly increased association with NIHL susceptibility than the TT carriers. There was a higher risk of NIHL in the XPO5 gene rs7755135 CC carriers than in the TT carriers. No statistically significant correlation was obtained with respect to SNPrs1106841. Functional experiments showed that the rs 11077 change might inhibit the interaction between miRNAs (miRNA-4763-5p, miRNA-5002-3p, and miRNA-617) and XPO5, with rs11077G allele resulting in overexpression of XPO5. Conclusion. The genetic polymorphism, rs11077, within XPO5 is associated with the risk of noise-induced hearing loss in a Chinese population.
\end{abstract}

\section{Introduction}

Noise-induced hearing loss (NIHL) refers to progressive sensorineural hearing impairment caused by patients exposed to a noisy environment. NIHL has become a major public health problem with industrialization, the increase in social noise, and the prolongation of life expectancy. Based on the global disease burden report issued by the WHO in 2005, occupational noise-associated hearing loss accounts for $16 \%$ of adult hearing loss worldwide, which is about 4 million disability-adjusted life years (DALYs) [1]. There are greater than 10 million workers employed in high-noise environments in China, of which at least $10 \%$ have different levels of hearing loss. Although environmental factors play a vital part in the development of NIHL, population epidemiologic studies have shown that, with the exception of the influence of other confounding factors, hereditary factors account for up to $50 \%$ of the variability in hearing loss after noise exposure [2,3].

MicroRNAs (miRNAs) are highly-conserved, endogenous noncoding single-stranded RNAs with posttranscriptional regulatory functions that are found in eukaryotes and consist of approximately 22 nucleotides (nt) [4,5]. Greater than 700 kinds of miRNAs have been identified in humans and regulate at least $30 \%$ of protein-coding gene expression $[6,7]$. miRNAs are transcribed into primary transcripts of miRNAs (pri-miRNAs) in the nucleus by RNA polymerase II and then further assimilated by RNase III Drosha to form a 
hairpin structure of approximately 60- to 70-nt precursor miRNAs (pre-miRNAs) [8,9]. Pre-miRNAs are transported from the nucleus to the cytoplasm under the synergistic action of transport receptor exportin-5 (XPO5). It is believed that single-nucleotide polymorphisms (SNPs) exist in the binding sites of the gene encoding miRNAs and related target genes, which can directly or indirectly affect gene expression and protein function [10].

Recent studies have confirmed that the abnormal expression of miRNAs is related to many diseases, including auditory diseases [11]. There is increasing evidence that the imbalance of miRNAs in NIHL affects the expression of target genes and further affects the necessary cellular processes, including cell metabolism, proliferation, differentiation, and apoptosis [12,13]. Compared with the control group, the expression of miRNA-24, miRNA-185-5p, and miRNA-451a increased significantly in the NIHL group [14]. Li et al. [15] reported that the serum miRNA-1229-5p level of male workers suffering from NIHL was significantly higher than the control group.

XPO5 exists in the nuclear membrane and participates in the transport of pre-miRNAs. Previous results have indicated that overexpression of XPO5 enhances the activity of miRNAs, and under- or nonexpression of XPO5 results in a decrease in the nuclear output of pre-miRNAs [16,17]. SNPs associated with miRNAs in the XPO5 $3^{\prime}$ untranslated region ( $3^{\prime} \mathrm{UTR}$ ) affect the risk of disease in the synthesis pathway of miRNAs [18]. Currently, there is a lack of research on the relationship between XPO5 and the risk of NIHL development; however, several studies have demonstrated that the transporter, XPO5, is involved in the miRNA pathway. The structural changes of XPO5 may cause abnormal expression of the miRNA, leading to tumorigenesis [19-21], which may also be the case in the hearing system. Based on bioinformatics prediction and statistical analysis, we found that SNPs in XPO5 may play a potential role in NIHL. In this study, we focused on the SNPs located in the binding region of miRNAs and XPO5. For significant SNPs, we performed functional validation to evaluate the potential function of these SNPs on XPO5.

\section{Materials and Methods}

2.1. Study Population Collection. In the current study, a total of 1040 cases and 1060 controls were recruited from the Yizheng Branch of the SAIC Volkswagen Automobile Co., Ltd. The subjects were engaged in steady-state noise work for a long time, and the exposure period of noise was not less than 1year. Workers exposed to noise did not have any history of disease or current illness that might affect their hearing, nor of the long-term use of ototoxic drugs. The definition of hearing loss was as follows: the subject's audiogram falls at high frequencies; the average hearing threshold of high frequencies in both ears and the better unilateral ear are $>25 \mathrm{~dB}$; and the high frequencies are greater than the low frequencies. Normal hearing refers to a subject's high and low frequency threshold $\leq 25 \mathrm{~dB}$. NIHL cases and controls were matched, including age, gender, smoking status, and noise exposure time. This study was approved by the Ethics Committee of Jiangsu Centers for Disease Control and Prevention, and all of the subjects signed the informed consent in person. Considering the use of data analysis, the private information of subjects involved in the study was encrypted.

2.2. SNP Selection. SNP loci located in the XPO5 gene were found from NCBIdbSNP (http://www.ncbi.nlm.nih.gov/ SNP) and ENSEMBL (http://www.ensembl.org/). The principles for screening SNPs are indicated as follows: (a) XPO5 binding site; (b) minor allele frequency (MAF) $>0.05$; (c) $p$ of Hardy-Weinberg equilibrium (HWE) $>0.05$; and (d) linkage disequilibrium (LD) of $r^{2}>0.8$. Four SNPs (rs2257082, rs11077, rs7755135, and rs1106841) were selected as candidate SNPs because the target gene (XPO5) was associated with the pathogenesis of NIHL.

2.3. Genetic Analysis. Peripheral blood samples from the subjects were stored in Vacutainers ${ }^{\circledR}$ containing the anticoagulant, (ethylenediaminetetraacetic acid) EDTA. The total DNA template was extracted using a DNA extraction kit (QIAGEN, Duesseldorf, Germany). The concentration and purity of the extracted DNA were determined using an ultraviolet spectrophotometer (NanoDrop ND-1000; Thermo Scientific, Wilmington, DE, USA). Based on the NCBIdbSNP database, the primers of the SNP locus were designed. The genomic DNA was amplified using an ABI7900HT real-time PCR system (Applied Biosystems, Foster City, CA,USA) at $94^{\circ} \mathrm{C}$ for $5 \mathrm{~min}$, followed by 40 cycles at $94^{\circ} \mathrm{C}$ for $20 \mathrm{~s}, 56^{\circ} \mathrm{C}$ for $30 \mathrm{~s}, 72^{\circ} \mathrm{C}$ for $30 \mathrm{~s}$, and $72^{\circ} \mathrm{C}$ for $7 \mathrm{~min}$. Detection of gene polymorphisms was controlled by ABITaqManSNP genotyping assays (Applied Biosystems, Foster City, CA, USA). Five percent samples were randomly sampled for quality control of duplicate genotyping, and the reproducibility of SNPs was $100 \%$.

2.4. Plasmid Construction. Human miRNA-4763-5p, miRNA-5002-3p, and miRNA-617 were cloned into the expression vector pcDNA3.1(+) to generate stably-transfected human embryonic kidney cell lines (HEK293T) using forward primers (GAATCTGGTCACCTGATGGGA) and reverse primers (GTGCCTGAGTGGACCTTGAG). The plasmid containing the sequence of the wild-type or mutant miRNA-4763-5p, miRNA-5002-3p, or miRNA-617 binding XPO5, was cloned into the luciferase reporter vector (pGL3CMV-LUC-MCS). The successfully constructed expression vector was inoculated into LB (Luria-Bertani) medium and cultured on a shaking table $(220 \mathrm{rpm})$ at $37^{\circ} \mathrm{C}$ for $24 \mathrm{~h}$. The plasmids were extracted and sequenced using a high-purity plasmid extraction kit (QIAGEN).

2.5. Reagents, Cell Culture, and Transfection. The HEK293T cell lines used in this study were obtained from Novobio Scientific (Shanghai, China). All cells were preserved in Dulbecco's Modified Eagle's medium (DEME) fortified with $10 \%$ fetal bovine serum (FBS) and placed in a humidified atmosphere with $5 \% \mathrm{CO}_{2}$ at $37^{\circ} \mathrm{C}$. miRNA-4763- 
5p mimics, miRNA-5002-3p mimics, miRNA-617 mimics, and pRL-TK (internal reference) were purchased from Genomeditech (Shanghai, China). The transfection experiment was carried out when the cells reached 70\%-80\% confluence. The plasmid transfection was conducted on the basis of the instructions of the manufacturer of Lipofectamine $2000^{\mathrm{TM}}$ (Invitrogen, Carlsbad, CA, USA).

2.6. Luciferase Reporter Assay. For luciferase analysis, HEK293T cells were inoculated into 24-well plates. Cells transfected with luciferase vectors containing T wild-type or G-mutant XPO5 fragments and miRNAs (miRNA-4763-5p, miRNA-5002-3p, and miRNA-617) were collected and cultured in medium and washed twice with phosphatebuffered saline (PBS). The cells were fully lysed by adding $100 \mu \mathrm{l}$ of $1 \times$ passive lysis buffer (PLB; Promega, Madison, WI, USA) to each well. Luciferase activity measurement was carried out on the basis of the operating instructions of the luciferase reporter assay system (Promega, Madison, WI, USA). The activity of Renilla luciferase was normalized to that of Firefly luciferase. All transfections were in triplicate.

2.7. Statistical Analysis. Frequencies of alleles and genotypes at SNP loci were obtained by direct counting. The association of genotype and gene frequency between the case and control groups was determined by a chi-square test. An unconditional univariate logistic regression model was used to analyze the odds ratios (ORs) and their 95\% confidence intervals (95\% CIs) and to evaluate the correlation between the genotype of miR-SNPs and the occurrence of NIHL. Values are shown as the mean \pm standard deviation. Differences were analyzed by SPSS (version 19; IBM, Armonk, NY, USA), and correction of the haplotype $p$ value (Pc) was done using the Sidak, Holm's correction, and the results were regarded as statistically significant at $p<0.05$.

\section{Results}

3.1. Characteristics and Clinical Features of Study Participants. A total of 2100 participants were recruited (1040 cases and 1060 controls). Detailed demographic data of the individuals are shown in Table 1 . Controls were matched with NIHL cases in the distribution of age $(p=0.537)$, gender $(p=0.074)$, cigarette smoking $(p=0.448)$, alcohol consumption $(p=0.374)$, duration of noise exposure $(p=0.511)$, and intensity of noise exposure $(p=0.325)$. A higher high-frequency hearing threshold shift was observed in cases $((40.73 \pm 13.40) \mathrm{dB})$ compared to controls $(16.63 \pm 4.75 \mathrm{~dB})$. There were significant differences in the high-frequency hearing threshold between the two groups $(p<0.001)$.

3.2. Association between the XPO5 Gene Polymorphism and NIHL Susceptibility. The characteristics of selected SNPs with an MAF $>0.05$ are listed in Table 2. The XPO5 loci rs2257082, rs11077, rs7755135, and rs1106841 were consistent with the Hardy-Weinberg equilibrium (HWE; $p>0.05)$ in the control group; the minimum $p$ value of HWE was 0.15. Multivariate logistic regression analysis showed that the rs2257082, rs11077, and rs7755135 loci, but not rs1106841, of the XPO5 gene were significantly associated with NIHL after excluding the potential confounders (age, gender, cigarette smoking, and alcohol consumption, duration of noise exposure, and intensity of noise exposure). SPSS 19.0 was used for statistical analysis.

As illustrated in Table 3, the rs2257082 AG/GG carriers showed an increased risk of NIHL compared to the AA carriers in the dominant model (adjusted OR $=1.55,95 \% \mathrm{CI}$ : $1.23-1.95, p<0.001)$. The rs11077TG/GG carriers had a significantly increased association with NIHL susceptibility than TT carriers in the dominant (adjusted OR $=1.93,95 \%$ CI: $1.48-2.52, p<0.001$ ) and recessive models (adjusted $\mathrm{OR}=3.03$, 95\% CI: 1.19-7.76, $p<0.001)$. There was a higher risk of NIHL in the XPO5 gene rs7755135TT carriers than CC carriers in the codominant (adjusted OR $=2.70,95 \% \mathrm{CI}$ : 1.53-4.77, $p<0.001$ ) and recessive models (adjusted $\mathrm{OR}=2.42$, 95\% CI: $1.40-4.19, p<0.001)$. No statistically significant correlation was obtained for SNPrs1106841 in any of the models (codominant, dominant, or recessive models). In the allele model, the rs11077G carriers (adjusted $\mathrm{OR}=1.63,95 \% \mathrm{CI}: 1.30-2.03)$ had a significantly higher risk for NIHL $(p<0.001)$ and rs7755135T carriers (adjusted $\mathrm{OR}=1.12,95 \% \mathrm{CI}: 1.01-1.39)$ had a significantly higher risk for NIHL $(p=0.037)$. The results indicated that XPO5SNPrs2257082, rs11077, and rs7755135 may have a connection to NIHL.

3.3. Analysis of High-Frequency Hearing Threshold Shift (HFTS) in Selected SNPs. The data of all subjects in Figure 1 described that the HFTS of the XPO5rs2257082 AA genotype was mainly in the range of $28.48 \pm 17.62 \mathrm{~dB}$, and those of the $A G$ and $G G$ genotypes were in the range of $27.76 \pm 14.24 \mathrm{~dB}$ and $31.64 \pm 15.34 \mathrm{~dB}$, respectively. The HFTS in the GG genotypes of rs2257082 were significantly higher than in the AA genotypes $(p=0.006)$. For rs11077, the TT genotype was mainly in the range of $27.96 \pm 15.77 \mathrm{~dB}$, and the TG and GG genotypes were $30.96 \pm 13.98 \mathrm{~dB}$ and $41.91 \pm 21.16 \mathrm{~dB}$, respectively. The HFTS in the rs11077GG and TG genotype subjects were significantly higher than in the TT genotypes $(p<0.001$ and $p=0.0015$, respectively). The rs7755135 CC genotype was mainly in the range of $28.05 \pm 16.28 \mathrm{~dB}$, while the CT and TT genotypes were in the range of $28.90 \pm 13.47 \mathrm{~dB}$ and $37.27 \pm 18.12 \mathrm{~dB}$, respectively. The TT genotype exhibited a significantly greater HFTS risk compared with the CC and CT genotypes $(p<0.001$ and $p<0.001$, respectively); however, the HFTS of the AA, AC, and CC genotypes of rs1106841 were mainly $29.01 \pm 17.10 \mathrm{~dB}, 27.85 \pm 14.13 \mathrm{~dB}$, and $29.87 \pm 15.31 \mathrm{~dB}$, respectively. There were no significant differences in HFTS among the AA, AC, and CC genotypes $(p=0.106$, $p=0.076$, and $p=0.520$, respectively).

3.4. Analysis of SNP (rs2257082, rs11077, rs7755135, and rs1106841) Haplotype Distribution. Figure 2 showed that the linkage disequilibrium of XPO5rs2257082, rs11077, 
TABle 1: Demographic characteristics and clinical features.

\begin{tabular}{|c|c|c|c|c|c|}
\hline \multirow{2}{*}{ Variables } & \multicolumn{2}{|c|}{ Cases $(n=1040)$} & \multicolumn{2}{|c|}{ Controls $(n=1060)$} & \multirow[b]{2}{*}{$p$} \\
\hline & $n$ & $\%$ & $n$ & $\%$ & \\
\hline Age (years), mean $\pm \mathrm{SD}$ & \multicolumn{2}{|c|}{$39.50 \pm 8.52$} & \multicolumn{2}{|c|}{$39.27 \pm 8.34$} & 0.537 \\
\hline Sex & & & & & 0.074 \\
\hline Male & 978 & 94.04 & 980 & 92.45 & \\
\hline Female & 62 & 5.96 & 80 & 7.55 & \\
\hline Smoking & & & & & 0.448 \\
\hline Now & 533 & 52.46 & 515 & 49.71 & \\
\hline Ever & 68 & 6.69 & 76 & 7.34 & \\
\hline Never & 415 & 40.85 & 445 & 42.95 & \\
\hline Drinking & & & & & 0.374 \\
\hline Now & 229 & 22.76 & 265 & 25.33 & \\
\hline Ever & 214 & 21.27 & 221 & 21.13 & \\
\hline Never & 563 & 55.96 & 560 & 53.54 & \\
\hline Work time with noise (years), mean \pm SD & & & & & 0.511 \\
\hline Exposure level with noise $(\mathrm{dB})$, mean $\pm \mathrm{SD}$ & & & & & 0.325 \\
\hline High-frequency hearing threshold $(\mathrm{dB})$, mean $\pm \mathrm{SD}$ & & & & & $<0.001$ \\
\hline
\end{tabular}

TABLE 2: General information of selected SNPs and Hardy-Weinberg test.

\begin{tabular}{|c|c|c|c|c|c|c|c|}
\hline \multirow{2}{*}{ SNP } & \multirow{2}{*}{ Chromosome } & \multirow{2}{*}{$\begin{array}{c}\text { Functional } \\
\text { consequence }\end{array}$} & \multicolumn{3}{|c|}{ MAF } & \multicolumn{2}{|l|}{ Regulome DB } \\
\hline & & & Control & Database $^{\mathrm{a}}$ & $p^{b}$ & Function annotation & Score \\
\hline rs2257082 & $6: 43492578$ & $\begin{array}{l}\text { Synonymous } \\
\text { variant }\end{array}$ & 0.97 & 0.32 & 0.75 & $\begin{array}{l}\text { Protein binding; chromatin structure; histone } \\
\text { modifications }\end{array}$ & 4 \\
\hline rs11077 & $6: 43490947$ & $\begin{array}{l}3 \text { prime UTR } \\
\text { variant }\end{array}$ & 0.73 & 0.40 & 0.15 & $\begin{array}{l}\text { Protein binding; chromatin structure; histone } \\
\text { modifications }\end{array}$ & 4 \\
\hline rs7755135 & $6: 43490809$ & $\begin{array}{l}3 \text { prime UTR } \\
\text { variant }\end{array}$ & 0.67 & 0.22 & 0.61 & $\begin{array}{l}\text { Protein binding; single nucleotides; chromatin } \\
\text { structure; histone modifications }\end{array}$ & 1f \\
\hline rs1106841 & $6: 43496662$ & $\begin{array}{l}\text { Splice region } \\
\text { variant }\end{array}$ & 0.87 & 0.39 & 0.88 & Motifs; chromatin structure; histone modifications & 5 \\
\hline
\end{tabular}

${ }^{a}$ Data from NCBIdbSNP. ${ }^{b} p$ value of Hardy-Weinberg test.

rs7755135, and rs1106841. Table 4 summarizes the haplotype frequencies of SNPs analyzed between NIHL cases and control groups. Five common haplotypes (frequency $>3 \%$ ) were selected from four SNPs, which accounted for $90 \%$ of haplotype variation. The GGTA and GTCC haplotypes (rs2257082-rs11077-rs7755135-rs1106841) were associated with an increased risk of NIHL $(\mathrm{OR}=1.54,95 \% \mathrm{CI}$ : $1.30-1.1 .94, \quad p<0.001 ; \quad \mathrm{OR}=1.46, \quad 95 \% \quad \mathrm{CI}: 1.18-1.81$, $p<0.001)$. The GTCA haplotype was associated with a decreased risk of NIHL (OR $=0.81,95 \%$ CI: 0.68-0.96, $p=0.02)$.

\subsection{Predicted miRNAs That Potentially Bind to XPO5rs11077.} To demonstrate whether or not the rs11077SNP affects the prediction of miRNA binding to XPO5, we performed a bioinformatics analysis of XPO5. The results indicated that $X P O 5$ rs 11077 is located in a potential binding region for incorporation of miRNAs, including miRNA-4763-5p, miRNA-5002-3p, and miRNA-617. XPO5 contains the binding site of predicted miRNAs, as shown in Figure 3.

3.6. SNPs Interfered with the Interaction between miRNA (miRNA-4763-5p, miRNA-5002-3p, and miRNA-617) and XPO5. Transient transfection was carried out in vitro, and the expression of related activities was analyzed and measured by the dual-luciferase reporting system to illustrate whether or not the SNPrs11077T $>$ G variant affected the binding of XPO5 to miRNAs (miRNA-4763-5p, miRNA5002-3p, and miRNA-617). Figure 4 indicates that constructs containing the $\mathrm{G}$ allele of XPO5 combined with miRNA-4763-5p, miRNA-5002-3p, and miRNA-617 mimics significantly increased luciferase activity compared with the $\mathrm{T}$ allele in HEK293T $(p<0.01, p<0.05$, and $p<0.01$, respectively). These data suggested that miRNA4763-5p, miRNA-5002-3p, and miRNA-617 may directly target XPO5 with the rs $11077 \mathrm{G}$ allele.

\section{Discussion}

NIHL is one of the most common occupational diseases that seriously affect human health [11]. Globally, the incidence of NIHL is on the rise. The pathogenesis of NIHL has not been fully clarified [22]. An etiologic investigation showed that chronic ear diseases, alcohol consumption, smoking, and occupational factors are related to the occurrence and development of NIHL, but different individuals vary in their sensitivity to individual causes.

Currently, studies on miRNAs and hearing loss have demonstrated that these miRNAs can be used as potential biomarkers to indicate NIHL $[15,23]$. In a study involving miRNA-15a-1 and miRNA-18a in the development of the 
TABLE 3: Distribution of four polymorphisms and the association with NIHL.

\begin{tabular}{|c|c|c|c|c|c|c|c|c|}
\hline Genetic models & Genotypes & Cases $n=1040$ & Controls $n=1060$ & $p^{\mathrm{a}}$ & Adjusted OR $(95 \% \mathrm{CI})^{\mathrm{a}}$ & Holm & SidakSS & SidakSD \\
\hline$r s 2257082$ & & $n=1030$ & $n=1053$ & & & & & \\
\hline \multirow{3}{*}{ Codominant } & AA & 324 & 380 & & $1.00(\mathrm{ref})$ & & & \\
\hline & AG & 546 & 515 & $2.69 E-4$ & $1.54(1.22-1.94)$ & & & \\
\hline & GG & 160 & 158 & $1.51 E-3$ & $1.73(1.23-2.44)$ & & & \\
\hline \multirow{2}{*}{ Dominant } & $\mathrm{AA}$ & 324 & 380 & & $1.00(\mathrm{ref})$ & & & \\
\hline & AG/GG & 706 & 673 & $2.09 E-4$ & $1.55(1.23-1.95)$ & & & \\
\hline \multirow{2}{*}{ Recessive } & $\mathrm{AA} / \mathrm{AG}$ & 870 & 895 & & $1.00(\mathrm{ref})$ & & & \\
\hline & GG & 160 & 158 & 0.26 & $1.16(0.89-1.51)$ & & & \\
\hline \multirow{3}{*}{$\begin{array}{l}\text { Alleles } \\
\text { rs11077 }\end{array}$} & $\mathrm{A}$ & 1194 & 1275 & & $1.00(\mathrm{ref})$ & & & \\
\hline & G & 866 & 831 & 0.09 & $1.11(0.98-1.26)$ & 0.18 & 0.314 & 0.172 \\
\hline & & $n=1036$ & $n=1056$ & & & & & \\
\hline \multirow{3}{*}{ Codominant } & TT & 835 & 921 & & $1.00(\mathrm{ref})$ & & & \\
\hline & TG & 186 & 129 & $8 E-6$ & $1.85(1.41-2.43)$ & & & \\
\hline & GG & 15 & 6 & $7.1 E-3$ & $3.66(1.42-9.41)$ & & & \\
\hline \multirow{2}{*}{ Dominant } & $\mathrm{TT}$ & 835 & 921 & & $1.00(\mathrm{ref})$ & & & \\
\hline & TG/GG & 201 & 135 & $1 E-6$ & $1.93(1.48-2.52)$ & & & \\
\hline \multirow{2}{*}{ Recessive } & TT/TG & 1021 & 1050 & & $1.00(\mathrm{ref})$ & & & \\
\hline & GG & 15 & 6 & $2.04 E-2$ & $3.03(1.19-7.76)$ & & & \\
\hline \multirow{2}{*}{ Alleles } & $\mathrm{T}$ & 1856 & 1971 & & $1.00(\mathrm{ref})$ & & & \\
\hline & G & 216 & 141 & $1.43 E-5$ & $1.63(1.30-2.03)$ & $5.71 E-5$ & $5.71 E-5$ & $5.71 E-5$ \\
\hline rs7755135 & & $n=1034$ & $n=1057$ & & & & & \\
\hline \multirow{3}{*}{ Codominant } & CC & 689 & 735 & & $1.00(\mathrm{ref})$ & & & \\
\hline & CT & 305 & 302 & 0.12 & $1.19(0.95-1.49)$ & & & \\
\hline & TT & 40 & 20 & $6.19 E-4$ & $2.70(1.53-4.77)$ & & & \\
\hline \multirow{2}{*}{ Dominant } & $\mathrm{CC}$ & 689 & 735 & & $1.00(\mathrm{ref})$ & & & \\
\hline & $\mathrm{CT} / \mathrm{TT}$ & 345 & 322 & 0.04 & $1.26(1.01-1.57)$ & & & \\
\hline \multirow{2}{*}{ Recessive } & $\mathrm{CC} / \mathrm{CT}$ & 994 & 1037 & & 1.00 (ref) & & & \\
\hline & $\mathrm{TT}$ & 40 & 20 & $1.65 E-3$ & $2.42(1.40-4.19)$ & & & \\
\hline \multirow{3}{*}{$\begin{array}{l}\text { Alleles } \\
\text { rs1106841 }\end{array}$} & $\mathrm{C}$ & 1683 & 1772 & & $1.00(\mathrm{ref})$ & & & \\
\hline & $\mathrm{T}$ & 385 & 342 & 0.037 & $1.12(1.01-1.39)$ & 0.112 & 0.141 & 0.108 \\
\hline & & $n=1035$ & $n=1052$ & & & & & \\
\hline \multirow{3}{*}{ Codominant } & AA & 467 & 491 & & 1.00 (ref) & & & \\
\hline & $\mathrm{AC}$ & 483 & 463 & 0.18 & $1.16(0.93-1.44)$ & & & \\
\hline & $\mathrm{CC}$ & 85 & 98 & 0.88 & $0.97(0.67-1.40)$ & & & \\
\hline \multirow{2}{*}{ Dominant } & $\mathrm{AA}$ & 467 & 491 & & 1.00 (ref) & & & \\
\hline & $\mathrm{AC} / \mathrm{CC}$ & 568 & 561 & 0.22 & $1.15(0.92-1.42)$ & & & \\
\hline \multirow{2}{*}{ Recessive } & $\mathrm{AA} / \mathrm{AC}$ & 950 & 954 & & $1.00(\mathrm{ref})$ & & & \\
\hline & CC & 85 & 98 & 0.35 & $0.86(0.62-1.18)$ & & & \\
\hline \multirow{2}{*}{ Alleles } & $\mathrm{A}$ & 1417 & 1445 & & 1.00 (ref) & & & \\
\hline & $\mathrm{C}$ & 653 & 659 & 0.88 & $1.01(0.89-1.15)$ & 0.875 & 0.999 & 0.875 \\
\hline
\end{tabular}

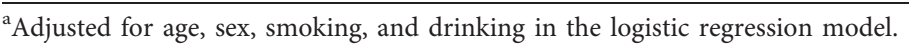

ear structure of zebrafish, it was found that the number of hair cells in the deformed body decreased, and the inner ear structure was abnormal [24]. MiR-34 mediates hearing impairment associated with cell death in the inner ear of a mouse model [25]. XPO5 is related to the nuclear output of miRNAs [26]. A synergistic effect exists between Ran-GTP and transport receptor $\mathrm{XPO5}$, which transports pre-miRNAs from the nucleus to the cytoplasm [27,28]. After digestion and double helix unwinding, pre-miRNAs bind with the RNA-induced silencing complex (RISC), which contains GEMIN3 and GEMIN4, to synthesize RISC-miRNA complexes [20]. The binding of RISC to the specific sequence of the $3^{\prime}$ UTR of target mRNAs results in the inhibition of the cleavage or translation of the mRNA, which interferes with the protein synthesis of the target gene at the posttranscriptional level $[29,30]$. Reducing the expression of XPO5 can decrease the expression of miRNAs, which may lead to the occurrence and development of hearing loss.

Considering the effect of age on hearing, we matched people in our study. Mizoue et al. [31] have proved that smoking can increase the risk of NIHL. To eliminate the interference of cigarette smoking, the matching included cigarette smoking. When binding of miRNAs to target sequences occurs at or near the miRNA junction in the $3^{\prime} U T R$, the SNPs have an effect by establishing or eliminating the binding sites of miRNAs in target genes, thus losing the original regulatory function and producing significant genetic effects. Accordingly, we systematically investigated the 


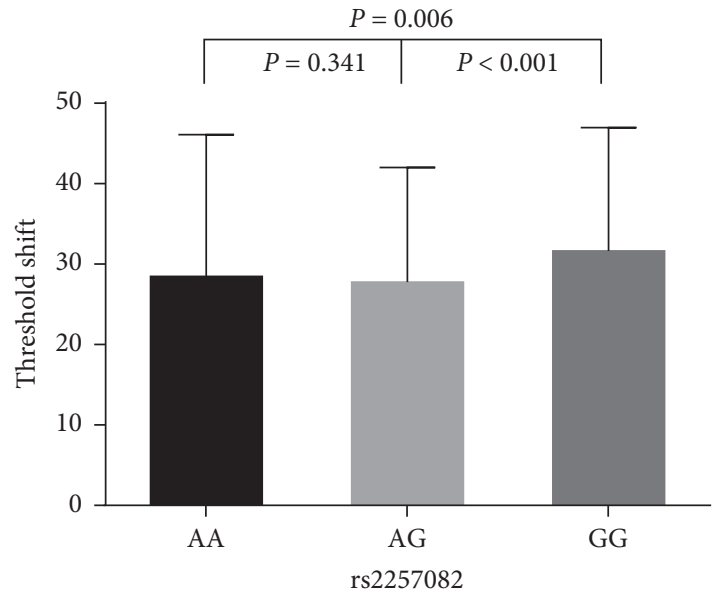

(a)

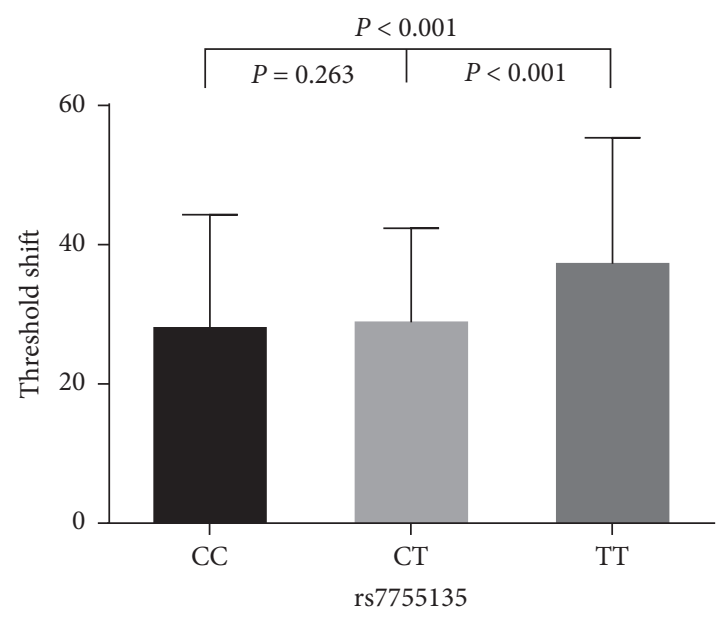

(c)

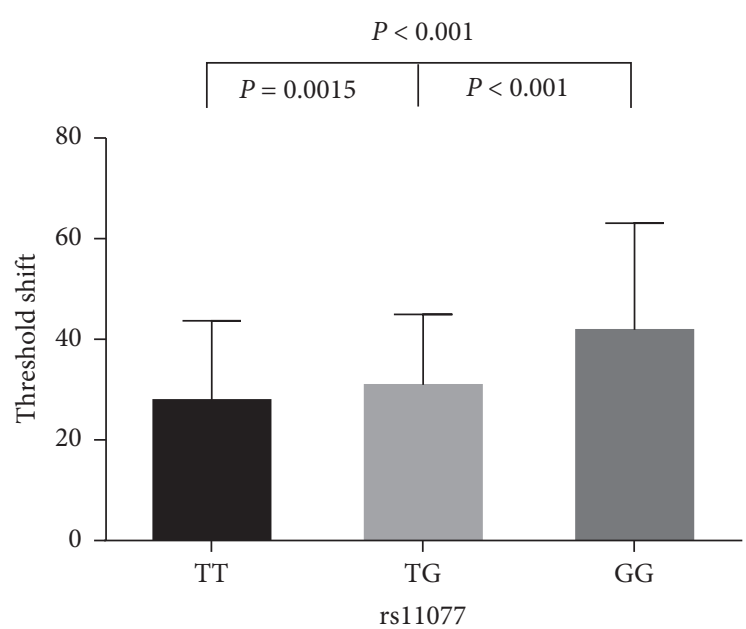

(b)

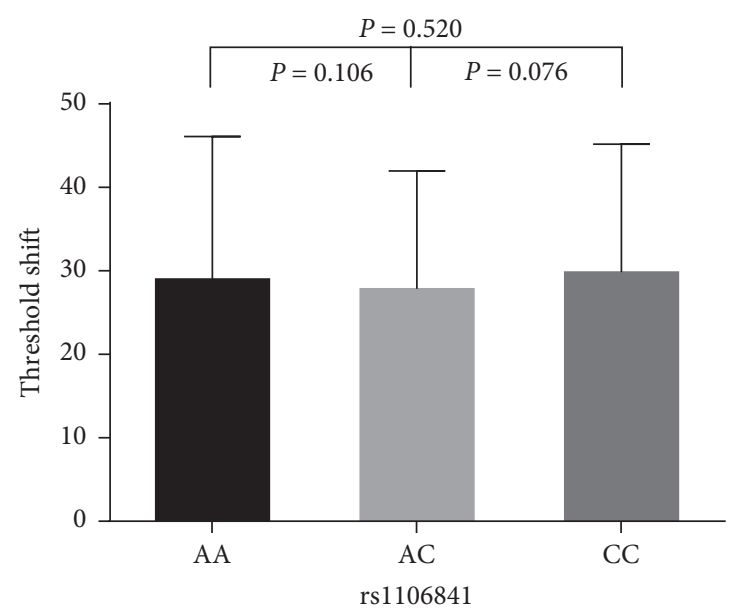

(d)

FIGURE 1: Comparison of high-frequency hearing threshold shift of four SNPs. Comparison of high-frequency hearing threshold shift of rs2257082, rs11077, rs7755135, and rs1106841 genotypes in all subjects. Data are presented as mean \pm SD, followed by analysis by ANOV. NS: no statistical significance.

potential correlation between the genetic polymorphism of XPO5 and NIHL in the Chinese population and discovered SNPs (rs2257082, rs11077, and rs7755135) in the XPO5 gene. Our data showed that the frequencies of the rs2257082GG, rs11077GG, and rs7755135TT of the XPO5 gene were significantly increased in NIHL cases compared to the control group. Therefore, we speculated that the rs2257082, rs11077, and rs7755135 loci SNPs of the XPO5 were associated with NIHL risk. Haploid analysis revealed that the GGTA and GTCC haplotypes (rs2257082-rs11077rs7755135-rs1106841) increased the risk of NIHL, and the GTCA haplotype was associated with a decreased risk of NIHL. The results support our hypothesis that XPO5 polymorphism may be related to NIHL susceptibility.

miRNAs can degrade or inhibit protein translation by means of complete or incomplete complementary pairing with target gene mRNA. Therefore, miRNAs play a significant role in posttranscriptional regulation of gene expression. The mutation of the binding site of the target gene of miRNAs will affect the biosynthesis or biological function of
miRNAs, which will lead to the disorder of cellular function and eventually result in the occurrence of diseases. SNPs can have a profound impact on miRNA function, including transcription, maturation, and target specificity [32], and it can also affect the occurrence of NIHL [33]. Recent studies have shown that SNPrs11077 in XPO5 gene is related to the risk of esophageal cancer, colorectal cancer, and renal cancer [34-36]. The A $>\mathrm{C}$ polymorphism of XPO5 gene will reduce the risk of CAD (coronary artery disease), which may be due to the influence on the expression of mature miRNAs and their gene function [37]. At the same time, functional SNPs in miRNA biogenetic pathway genes have been confirmed to be related to the increased risk of NIHL $[33,38]$. Based on all the above studies, we analyzed the relationship between the functional sites of XPO5 of miRNA processing gene and NIHL, and its effect on miRNA expression. In this study, luciferase reporter assays preliminarily verified whether miRNA interacted with target gene XPO53'UTR and further determined the site of interaction between miRNA and

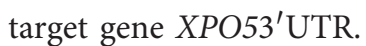




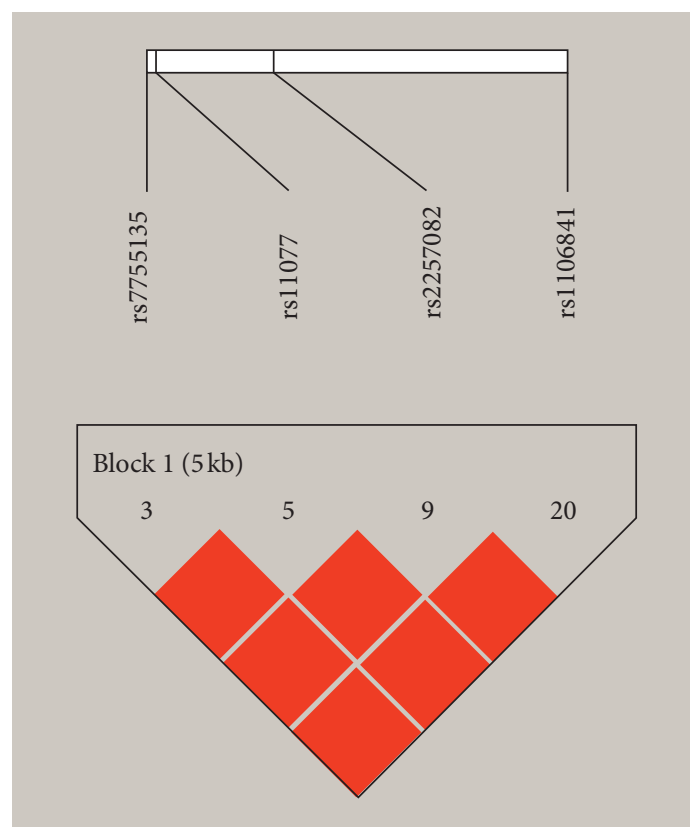

(a)

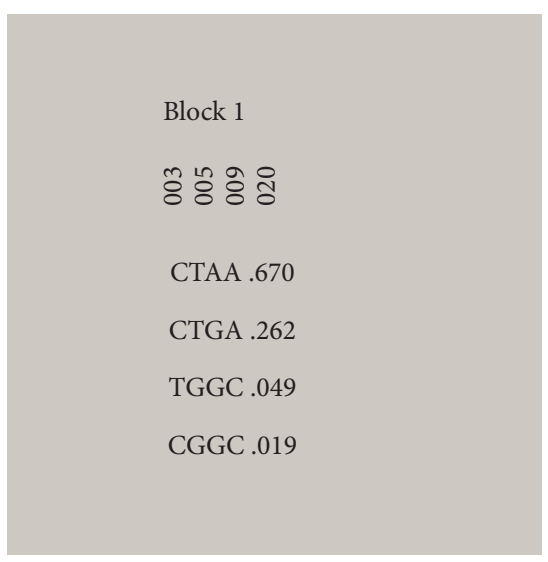

(b)

FIGURE 2: Reconstructed linkage disequilibrium (LD) plot for the four single-nucleotide polymorphisms (SNPs).

TABLE 4: Frequencies of inferred haplotypes among the cases and controls and their association with risk NIHL.

\begin{tabular}{lccccccccc}
\hline Haplotypes $^{\mathrm{a}}$ & Case (freq) & Control (freq) & Chi2 & Pearson's $p$ & OR $(95 \%$ CI) & Holm & SidakSS & SidakSD & Global $p$ value \\
\hline GGTA & $199(0.10)$ & $136(0.07)$ & 14.21 & $1.63 E-04$ & $1.54(1.30-1.94)$ & $8.16 E-04$ & $8.16 E-04$ & $8.16 E-04$ \\
GTTA & $166(0.08)$ & $196(0.09)$ & 2.13 & 0.14 & $0.85(0.69-1.06)$ & 0.17 & 0.54 & 0.17 \\
GTCA & $261(0.13)$ & $320(0.15)$ & 5.71 & 0.02 & $0.81(0.68-0.96)$ & 0.05 & 0.08 & 0.05 \\
GTCC & $222(0.11)$ & $160(0.08)$ & 12.41 & $4.28 E-04$ & $1.46(1.18-1.81)$ & 0.001 & 0.002 & 0.001 & 06 \\
ATCC & $1186(0.58)$ & $1264(0.61)$ & 2.93 & 0.09 & $0.90(0.79-1.02)$ & 0.17 & 0.37 & 0.17 & \\
\hline
\end{tabular}

${ }^{a}$ The alleles of the haplotypes were arrayed as rs2257082-rs11077-rs7755135-rs1106841. Haplotypes with frequency $<0.03$ are ignored.

H_XP05 3' UTR T allele 5' ...AGCACTAAAGACTTCCCAGCCC...3' | | | | | ||: || | hsa-miR-4763-5p $3^{\prime}$...TCGTCCTCCC-GACCCGTCCGC...5' | | | | | ||: || | |

H_XP05 3' UTR G allele 5' ...AGCACTAAAGACTGCCCAGCCC....3'

H_XP05 $3^{\prime}$ UTR T allele 5' ...AAGACTTCCCAGCCCTGGTCC.... $3^{\prime}$ | | $:||: \quad|: \quad::|||$ hsa-miRNA-5002-3p 3' ...TTCACCAGTCACTCCGTCAGT...5' || $:||: \quad|: \quad::|||$

H_XP05 3' UTR G allele 5' ...AAGACTGCCCAGCCCTGGTCC...3'

H_XP05 3' UTR T allele 5' ...AAAGACTTCCCAGCCCTGGTCC....3' || ||: : |: : ||| hsa-miR-617 3' ...CGGTGGAAGTTTACCCTTCAGA....3' ||||$::|: \quad:|||$

H_XP05 $3^{\prime}$ UTR G allele 5' ...AAAGACTGCCCAGCCCTGGTCC....3'

FIgure 3: Predicted binding of the candidate miRNAs (miR-47635p, miRNA-5002-3p, and miR-617) to SNPrs11077 of XPO53'UTR.

In addition to the interaction of multiple factors, rs11077 had the most significant correlation with NIHL compared with other loci. We selected rs11077 for functional verification. By virtue of the mutations located in the secondary structure of miRNA, as well as the number of mutants that can be detected, we investigated the possible effects of these mutations on target genes. First, we predicted potential targets based on bioinformatics programs (TargetScan, Microinspector, and miRanda). Meanwhile, considering the complementation, evolutionary conservation, accessibility, and thermal stability of the target gene locus (rs11077) to miRNA, we included these miRNAs (miRNA-4763-5p, miRNA-5002-3p, and miRNA-617) and conduct subsequent studies on luciferase activity. These miRNAs contain binding sites that match the seed region of XPO5 perfectly. Importantly, miRNA-4763 has recently been shown to contribute specifically to multidrug resistance in human cancer cells [32]. Wang et al. [33] reported that downregulation of miRNA-4763-3p expression increased the susceptibility to gastric cancer by targeting MDR. It was also predicted that the potential regulatory pathway of XPO5rs11077, and the binding ability of miRNA-4763-5p/miRNA-5002-3p would be affected when the $T$ wild-type allele mutated into the $G$ allele [34]. The results of luciferase reporter gene activity analysis in our study showed that the translation level of luciferase-UTR was controlled by miRNA-4763-5p, miRNA-5002-3p, and miRNA-617 compared with the T-allele miRNA-4763-5p and the miRNA-617, the $G$ allele 


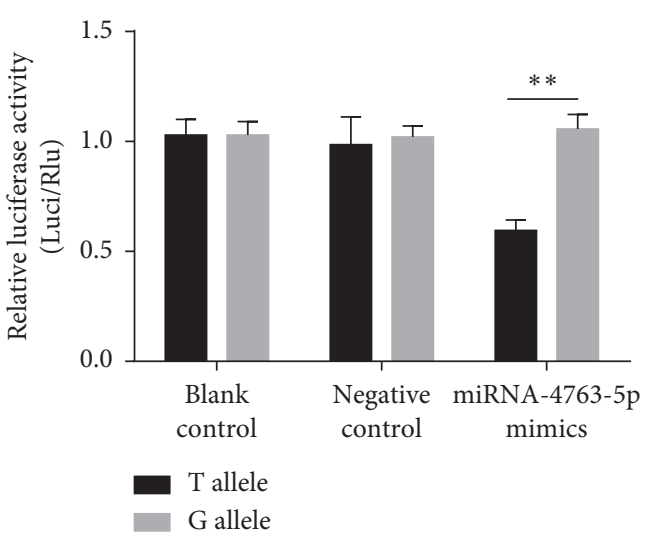

(a)

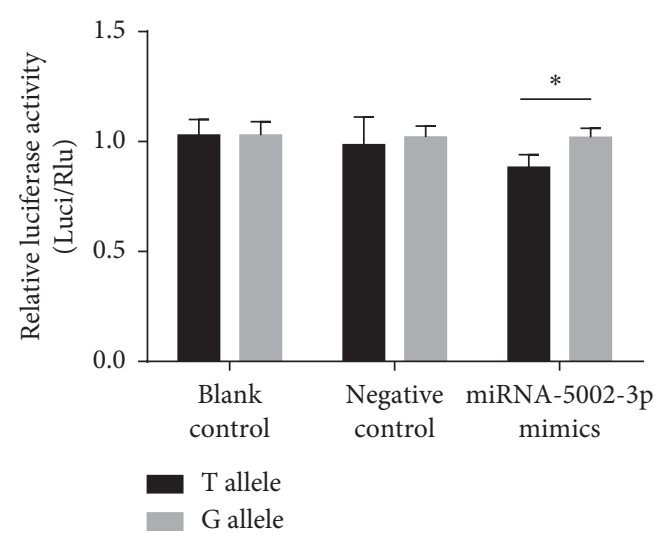

(b)

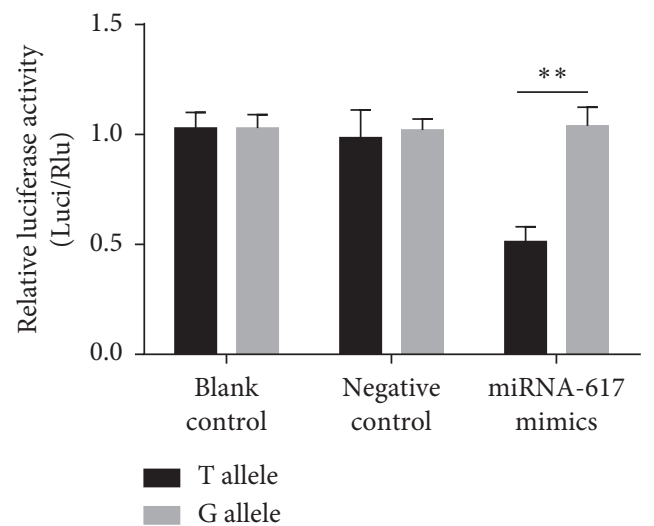

(c)

Figure 4: SNPrs11077T or G allele in the 3'-UTR of XPO5 differences with the presence or interference of miRNAs (miR-4763-5p, miRNA5002-3p, and miR-617) was analyzed by dual-luciferase report assay. Negative control means adding an unrelated sequence of miRNAs to the pGL3 vector coupled to the XPO53'-UTR regions (pGL3-3'UTR). Relative luciferase activity was expressed by the ratio of Firefly/Renilla activity. Data were presented as the mean \pm SD. ${ }^{*} p<0.05$ and ${ }^{* *} p<0.01$.

resulted in increased luciferase expression. This finding indicated that the mutation allele of rs11077 affected the binding of miRNAs (miRNA-4763-5p, miRNA-5002-3p, and miRNA-617) to XPO5.

\section{Conclusion}

In summary, our study provides evidence for the first time that the SNPrs11077G allele and the haplotype (rs2257082, rs11077, rs7755135, and rs1106841) had an association with the risk of NIHL in a Chinese population. It was also verified that the regulation of XPO5 expression by miRNA-4763-5p, miRNA-5002-3p, and miRNA-617 might be achieved by SNPrs11077.

\section{Data Availability}

The data sets supporting the results of this article are included within the article.

\section{Ethical Approval}

The participate's private information has been encrypted by Jiangsu Provincial Center for Disease Control and
Prevention (CDC). This study conforms to the Helsinki Declaration and was exempted from the review of institutional ethics by the Research Ethics Committee of Jiangsu CDC.

\section{Consent}

All presentations of reports must have consent for publication.

\section{Conflicts of Interest}

The authors declare that they have no conflicts of interests.

\section{Authors' Contributions}

Ning Wang and Boshen Wang designed and conducted the study. JiadiGuo and Suhao Zhang analyzed the data and generated the figures. Ning Wang, Boshen Wang, JiadiGuo, Suhao Zhang, Lei Han, Juan Zhang, and Baoli Zhu wrote the manuscript. Baoli Zhu revised the manuscript. All authors finally reviewed and approved the manuscript. 


\section{Acknowledgments}

The authors thank all of the participants. This study was supported by the Jiangsu Provincial Youth Medical Talent program (QNRC2016536), Preventive Medicine Foundation of Jiangsu (Y2015049), Six Talent Peaks Project in Jiangsu Province (WSW-017), Jiangsu Province's Outstanding Medical Academic Leader Program (CXTDA2017029).

\section{References}

[1] D. I. Nelson, R. Y. Nelson, M. Concha-Barrientos, and M. Fingerhut, "The global burden of occupational noise-induced hearing loss," American Journal of Industrial Medicine, vol. 48, no. 6, pp. 446-458, 2005.

[2] R. S. Abreu-Silva, D. Rincon, A. R. V. R. Horimoto et al., "The search of a genetic basis for noise-induced hearing loss (NIHL)," Annals of Human Biology, vol. 38, no. 2, pp. 210218, 2011.

[3] M. Sliwinska-Kowalska and A. Davis, "Noise-induced hearing loss," Noise and Health, vol. 14, no. 61, pp. 274-280, 2012.

[4] C. Sucharov, M. R. Bristow, and J. D. Port, "miRNAexpression in the failing human heart: functional correlates," Journal of Molecular and Cellular Cardiology, vol. 45, no. 2, pp. 185-192, 2008.

[5] R. C. Friedman, K. K. Farh, C. B. Burge, and D. P. Bartel, "Most mammalian mRNAs are conserved targets of microRNAs," Genome Research, vol. 19, no. 1, pp. 92-105, 2009.

[6] B. P. Lewis, C. B. Burge, and D. P. Bartel, "Conserved seed pairing, often flanked by adenosines, indicates that thousands of human genes are microRNA targets," Cell, vol. 120, no. 1, pp. 15-20, 2005.

[7] D. P. Bartel, "MicroRNAs: genomics, biogenesis, mechanism, and function," Cell, vol. 116, no. 2, pp. 281-297, 2004.

[8] J. Wen, Q. Gao, N. Wang et al., "Association of microRNArelated gene XPO5rs11077 polymorphism with susceptibility to thyroid cancer," Medicine, vol. 96, no. 14, Article ID e6351, 2017.

[9] G. Meister, M. Landthaler, Y. Dorsett, and T. Tuschl, "Sequence-specific inhibition of microRNA- and siRNA-induced RNA silencing," RNA, vol. 10, no. 3, pp. 544-550, 2004.

[10] K. Mochida, Y. Yamazaki, and Y. Ogihara, "Discrimination of homoeologous gene expression in hexaploid wheat by SNP analysis of contigs grouped from a large number of expressed sequence tags," Molecular Genetics and Genomics, vol. 270, no. 5, pp. 371-377, 2003.

[11] G. Solda, M. Robusto, P. Primignani et al., "A novel mutation within the MIR96 gene causes non-syndromic inherited hearing loss in an Italian family by altering pre-miRNA processing," Human Molecular Genetics, vol. 21, no. 3, pp. 577-585, 2012.

[12] V. Ambros, "The functions of animal microRNAs," Nature, vol. 431, no. 7006, pp. 350-355, 2004.

[13] J. Kim, F. Yao, Z. Xiao, Y. Sun, and L. Ma, "MicroRNAs and metastasis: small RNAs play big roles," Cancer and Metastasis Reviews, vol. 37, no. 1, pp. 5-15, 2018.

[14] L. Ding, J. Liu, H.-X. Shen et al., "Analysis of plasma microRNA expression profiles in male textile workers with noise-induced hearing loss," Hearing Research, vol. 333, pp. 275-282, 2016.

[15] Y. H. Li, Y. Yang, Y. T. Yan et al., "Analysis of serum microRNA expression in male workers with occupational noise-induced hearing loss," Brazilian Journal of Medical and Biological Research, vol. 51, no. 3, Article ID e6426, 2018.
[16] R. Yi, Y. Qin, I. G. Macara, and B. R. Cullen, "Exportin-5 mediates the nuclear export of pre-microRNAs and short hairpin RNAs," Genes \& Development, vol. 17, no. 24, pp. 3011-3016, 2003.

[17] E. Lund, S. Guttinger, A. Calado, J. E. Dahlberg, and U. Kutay, "Nuclear export of microRNA precursors," Science, vol. 303, no. 5654, pp. 95-98, 2004.

[18] Z. Guo, H. Wang, Y. Li, B. Li, C. Li, and C. Ding, "A microRNA-related single nucleotide polymorphism of the XPO5 gene is associated with survival of small cell lung cancer patients," Biomedical Reports, vol. 1, no. 4, pp. 545-548, 2013.

[19] Y. Li, X. Wang, B. He, H. Cai, and Y. Gao, "Downregulation and tumor-suppressive role of $\mathrm{XPO}_{5}$ in hepatocellular carcinoma," Molecular and Cellular Biochemistry, vol. 415, no. 12, pp. 197-205, 2016.

[20] M. CampayoA. Navarro et al., "A dual role for KRT81: a miRSNP associated with recurrence in non-small-cell lung cancer and a novel marker of squamous cell lung carcinoma," PLoS One, vol. 6, no. 7, Article ID e22509, 2011.

[21] C. Ding, C. Li, H. Wang, B. Li, and Z. Guo, "A miR-SNP of the XPO5 gene is associated with advanced non-small-cell lung cancer," OncoTargets and Therapy, vol. 6, pp. 877-881, 2013.

[22] P. M. Rabinowitz, "Noise-induced hearing loss," American Family Physician, vol. 61, no. 9, pp. 2749-2756, 2000.

[23] V. Miguel, J. Y. Cui, L. Daimiel et al., "The role of microRNAs in environmental risk factors, noise-induced hearing loss, and mental stress," Antioxid Redox Signaling, vol. 28, no. 9, pp. 773-796, 2018.

[24] L. M. Friedman, A. A. Dror, E. Mor et al., "MicroRNAs are essential for development and function of inner ear hair cells in vertebrates," Proceedings of the National Academy of Sciences, vol. 106, no. 19, pp. 7915-7920, 2009.

[25] L. Yu, H. Tang, X. Hua Jiang, L. Ling Tsang, Y. Wa Chung, and H. Chang Chan, "Involvement of calpain-I and microRNA34 in kanamycin-induced apoptosis of inner ear cells," Cell Biology International, vol. 34, no. 12, pp. 1219-1225, 2010.

[26] A. Mencia, S. Modamio-Hoybjor, N. Redshaw et al., "Mutations in the seed region of human miR-96 are responsible for nonsyndromic progressive hearing loss," Nature Genetics, vol. 41, no. 5, pp. 609-613, 2009.

[27] A. M. Brownawell and I. G. Macara, "Exportin-5, a novel karyopherin, mediates nuclear export of double-stranded RNA binding proteins," The Journal of Cell Biology, vol. 156, no. 1, pp. 53-64, 2002.

[28] M. T. Bohnsack, K. Czaplinski, and D. Gorlich, "Exportin 5 is a RanGTP-dependent dsRNA-binding protein that mediates nuclear export of pre-miRNAs," RNA, vol. 10, no. 2, pp. 185-191, 2004.

[29] L. Peters and G. Meister, "Argonaute proteins: mediators of RNA silencing," Molecular Cell, vol. 26, no. 5, pp. 611-623, 2007.

[30] A. J. Pratt and I. J. MacRae, "The RNA-induced silencing complex: a versatile gene-silencing machine," Journal of Biological Chemistry, vol. 284, no. 27, pp. 17897-17901, 2009.

[31] T. Mizoue, T. Miyamoto, and T. Shimizu, "Combined effect of smoking and occupational exposure to noise on hearing loss in steel factory workers," Occupational and Environmental Medicine, vol. 60, no. 1, pp. 56-59, 2003.

[32] A. Keller, T. Rounge, C. Backes et al., "Sources to variability in circulating human miRNA signatures," RNA Biology, vol. 14, no. 12, pp. 1791-1798, 2017.

[33] Y. Wu, J. Ni, M. Qi et al., "Associations of genetic variation in CASP3 gene with noise-induced hearing loss in a Chinese 
population: a case-control study," Environmental Health, vol. 16, no. 1, p. 78, 2017.

[34] M. A. Saunders, H. Liang, and W. H. Li, "Human polymorphism at microRNAs and microRNA target sites," Proceedings of the National Academy of Sciences, vol. 104, no. 9, pp. 3300-3305, 2007.

[35] S. Liu, J. An, J. Lin et al., "Single nucleotide polymorphisms of microRNA processing machinery genes and outcome of hepatocellular carcinoma," PLoS One, vol. 9, no. 3, Article ID e92791, 2014.

[36] C. F. de Larrea, A. Navarro, R. Tejero et al., "Impact of MiRSNPs on survival and progression in patients with multiple myeloma undergoing autologous stem cell transplantation," Clinical Cancer Research, vol. 18, no. 13, pp. 3697-3704, 2012.

[37] A. Borghini, S. Pulignani, A. Mercuri et al., "Influence of genetic polymorphisms in DICER and XPO5 genes on the risk of coronary artery disease and circulating levels of vascular miRNAs," Thrombosis Research, vol. 180, pp. 32-36, 2019.

[38] X. Zhang, Y. Liu, L. Zhang et al., "Associations of genetic variations in EYA4, GRHL2 and DFNA5 with noise-induced hearing loss in Chinese population: a case-control study," Environmental Health, vol. 14, no. 1, p. 77, 2015. 